Western University

Scholarship@Western

Brain and Mind Institute Researchers'

Publications

Brain and Mind Institute

$1-1-2014$

\title{
Observing object lifting errors modulates cortico-spinal excitability and improves object lifting performance.
}

\author{
Gavin Buckingham \\ Department of Psychology, School of Life Sciences, Heriot-Watt University, Edinburgh, UK; The Brain and \\ Mind Institute, The University of Western Ontario, Canada, g.buckingham@hw.ac.uk \\ Jeremy D Wong \\ The Brain and Mind Institute, The University of Western Ontario, Canada; The Department of Psychology, \\ The University of Western Ontario, Canada; The Graduate Program in Neuroscience, The University of \\ Western Ontario, Canada \\ Minnie Tang \\ The Department of Physiology and Pharmacology, The University of Western Ontario, Canada \\ Paul L Gribble \\ The Brain and Mind Institute, The University of Western Ontario, Canada; The Department of Psychology, \\ The University of Western Ontario, Canada; The Department of Physiology and Pharmacology, The \\ University of Western Ontario, Canada \\ Melvyn A Goodale \\ The Brain and Mind Institute, The University of Western Ontario, Canada; The Department of Psychology, \\ The University of Western Ontario, Canada
}

Follow this and additional works at: https://ir.lib.uwo.ca/brainpub

Part of the Neurosciences Commons, and the Psychology Commons

\section{Citation of this paper:}

Buckingham, Gavin; Wong, Jeremy D; Tang, Minnie; Gribble, Paul L; and Goodale, Melvyn A, "Observing object lifting errors modulates cortico-spinal excitability and improves object lifting performance." (2014). Brain and Mind Institute Researchers' Publications. 194.

https://ir.lib.uwo.ca/brainpub/194 


\title{
Research report
}

\section{Observing object lifting errors modulates cortico-spinal excitability and improves object lifting performance}

\author{
Gavin Buckingham ${ }^{a, b, *}$, Jeremy D. Wong ${ }^{b, c, d}$, Minnie Tang ${ }^{e}$, Paul L. Gribble ${ }^{b, c, e}$ and \\ Melvyn A. Goodale ${ }^{b, c}$ \\ ${ }^{a}$ Department of Psychology, School of Life Sciences, Heriot-Watt University, Edinburgh, UK \\ ${ }^{\mathrm{b}}$ The Brain and Mind Institute, The University of Western Ontario, Canada \\ ${ }^{\mathrm{c}}$ The Department of Psychology, The University of Western Ontario, Canada \\ $\mathrm{d}$ The Graduate Program in Neuroscience, The University of Western Ontario, Canada \\ ${ }^{\mathrm{e}}$ The Department of Physiology and Pharmacology, The University of Western Ontario, Canada
}

\section{A R T I C L E I N F O}

Article history:

Received 12 February 2013

Reviewed 26 April 2013

Revised 17 May 2013

Accepted 9 July 2013

Action editor Laurel Buxbaum

Published online 19 July 2013

\section{Keywords:}

Object lifting

Size-weight illusion

Action observation

Motor learning

Grip force error

\begin{abstract}
A B S T R A C T
Observing the actions of others has been shown to modulate cortico-spinal excitability and affect behaviour. However, the sensorimotor consequences of observing errors are not well understood. Here, participants watched actors lift identically weighted large and small cubes which typically elicit expectation-based fingertip force errors. One group of participants observed the standard overestimation and underestimation-style errors that characterise early lifts with these cubes (Error video - EV). Another group watched the same actors performing the well-adapted error-free lifts that characterise later, well-practiced lifts with these cubes (No error video - NEV). We then examined actual object lifting performance in the subjects who watched the EV and NEV. Despite having similar cognitive expectations and perceptions of heaviness, the group that watched novice lifters making errors themselves made fewer overestimation-style errors than those who watched the expert lifts. To determine how the observation of errors alters cortico-spinal excitability, we measured motor evoked potentials in separate group of participants while they passively observed these EV and NEV. Here, we noted a novel size-based modulation of cortico-spinal excitability when observing the expert lifts, which was eradicated when watching errors. Together, these findings suggest that individuals' sensorimotor systems are sensitive to the subtle visual differences between observing novice and expert performance.
\end{abstract}

(c) 2013 Elsevier Ltd. All rights reserved.

\section{Introduction}

Before picking up an object, individuals will implicitly estimate its weight based on its visual properties, and these expectations of heaviness drive the way that they lift objects.
This means that when lifting something for the first time, a lifter's fingertip forces reflect their initial predictions about an object's weight, rather than the actual mass of the object (Gordon, Forssberg, Johansson, \& Westling, 1991). The feedforward nature of human lifting behaviour often results in

\footnotetext{
* Corresponding author. David Brewster Building, Heriot-Watt University, Edinburgh EA14 4AS, United Kingdom.

E-mail addresses: g.buckingham@hw.ac.uk, gav.buckingham@gmail.com (G. Buckingham). 0010-9452/\$ - see front matter ๔ 2013 Elsevier Ltd. All rights reserved. 
grip and load force errors, which can be especially dramatic when objects have an unusual weight for their appearance (e.g., Buckingham, Cant, \& Goodale, 2009; Johansson \& Westling, 1988). These errors do not generally persist and individuals are rapidly able to overcome their expectations of heaviness, tuning their fingertip forces to the actual, rather than expected, weight of the object(s) being lifted (Flanagan \& Beltzner, 2000; Grandy \& Westwood, 2006; Mon-Williams \& Murray, 2000). In other words, when lifting objects repeatedly, individuals rapidly and implicitly learn to lift them with the appropriate level of grip and load forces for their actual weight.

Despite the widely held assumption that fingertip force adaptation is mediated solely by fast-adapting Type-2 afferents in the fingertips (Johansson \& Flanagan, 2009), it has recently been demonstrated that vision plays a crucial role in this form of motor learning. When they are deprived of vision individuals show deficits in their ability to correct their fingertip force errors, continually lifting objects with forces that reflect how heavy the objects look, rather than how heavy the objects actually are (Buckingham \& Goodale, 2010a; Buckingham, Ranger, \& Goodale, 2011). These findings indicate that individuals receive valuable information describing the direction and magnitude of a lifting error from visual kinematic cues.

Consistent with this proposal, a variety of studies have demonstrated that humans are surprisingly adept at acquiring useful information, such as object weight, from the observed visual kinematics of others' lifts (Bingham, 1987; Hamilton, Joyce, Flanagan, Frith, \& Wolpert, 2007). Not only are individuals able to use these kinematic cues, but there is emerging evidence that the link between acting and perceiving is an automatic one. Hamilton and colleagues (Hamilton, Wolpert, \& Frith, 2004) demonstrated that our perception of an actor's lift is modulated by the weight of an object the observer is holding (interestingly, in the opposite direction from what might be expected - holding a light box made the observed lift appear comparatively effortful, and vice versa). Furthermore, individuals implicitly use kinematic cues observed in other lifters when lifting objects which have an unpredictable weight (Meulenbroek, Bosga, Hulstijn, \& Miedl, 2007). Perhaps the strongest argument for an automatic link between visual kinematics and action production in the context of object lifting comes from a recent series of action observation studies showing that the sensorimotor system appears to encode the force requirements of an observed lift. Using transcranial magnetic stimulation (TMS) to evoke motor potentials (MEPs) in a passive observation task, Alaerts, Swinnen, and Wenderoth (2009) demonstrated that merely watching a video of someone else lifting a heavy object elicits a larger MEP than is elicited while watching a similar video of a lighter object. Subsequent studies have revealed that this force-related modulation of cortico-spinal excitability was caused by differences between the kinematics of the effortful (heavy objects) and easy (light objects) lifts, rather than semantic or material-based visual cues to object (Alaerts, Senot, et al., 2010; Alaerts, Swinnen, \& Wenderoth, 2010; Senot et al., 2011). The effects of observing the actions of others are not limited to the modulation of cortico-spinal excitability. A recent study has shown that the forces involved in lifting can be modulated by observing others, elegantly demonstrating that, compared to viewing an object being lightly touched, watching an actor firmly pinching a target object will increase the gripping force subsequently used to lift that object (Uçar \& Wenderoth, 2012).

These studies tend to be interpreted within the broader context of the putative human mirror neuron system (Gallese, Gernsbacher, Heyes, Hickok, \& Iacoboni, 2011; Mukamel, Ekstrom, Kaplan, Iacoboni, \& Fried, 2010). The overlapping neuronal populations and cortical regions in human and nonhuman primates has been taken by some as a mechanism for observational learning, by means of implicit neural simulation of the observed action (Calvo-Merino, Glaser, Grèzes, Passingham, \& Haggard, 2005; Jeannerod, 2001). However, the concept of mirror neurons, as typically discussed, offers no insight into how the sensorimotor system reacts to the observation of the commonplace errors that must drive motor learning. This question needs to be addressed at both the level of behaviour and cortico-spinal excitability. In terms of behaviour, it would presumably be maladaptive for the sensorimotor system to copy the motor output of an observed error. Although very few empirical studies have examined the consequences of error observation in any context whatsoever, some recent hints have emerged that individuals can improve their subsequent performance by observing errors. In Mattar and Gribble (2005), participants reached in a velocity-dependant force field toward a visual target - a task which normally requires a substantial amount of learning. They noted that after observing videos of others performing an aiming task, participants performing the same task learnt to overcome the dynamics of the force-field more rapidly. Furthermore, observing a different force-field from the one they eventually had to deal with substantially slowed their rate of adaptation, hinting at an automatic observational learning effect (see also Brown, Wilson, \& Gribble, 2009). Crucially, Brown and colleagues parametrically varied the degree of error in these videos, noting that participants were able to benefit more from observing larger errors than smaller ones (Brown, Wilson, Obhi, \& Gribble, 2010). This finding was, of course, not an unexpected result given that the correct performance in the task relied exclusively on vision, and the errors provide the only visual indications of the situational dynamics. This work does, however, provide some preliminary hints that there may be a specific and important role for error observation in subsequent behavioural outputs, leading us to predict that observing lifting errors will improve subsequent lifting performance more than observing well-practiced lifts. The role of errors in driving cortico-spinal excitability is less clear, with no work examining MEPs during the observation of motor errors. As there are indications that observing errors may help improve subsequent performance, it is possible that the errors are encoded by the sensorimotor system to drive the subsequent corrective behavioural response. If the lowlevel motor resonance within the sensorimotor system slavishly mimics what is observed, such a mirroring response would manifest as a large MEP for an overestimation of force and a small MEP for an underestimation of force. However, as errors appear to drive improved behaviour (i.e., in directional opposition to the initial error), the MEPs might in fact oppose the pattern of resonance normally evoked by lifting forces - a 
large MEP to counteract an erroneous underestimation of force and a small MEP to counteract an erroneous overestimation of force.

The goal of the current work was to examine the consequences of observing errors, within the simple motor learning framework of fingertip force adaptation during object lifting. To this end, we examined the sensorimotor consequences of watching the visual consequences of overestimations and underestimations of lifting forces (the error video - EV) as compared to well-adapted object lifting performance (the no error video - NEV) at the behavioural and cortico-spinal level. If errors are in fact crucial cues for observational learning, it is likely that observing them will (1) improve fingertip force adaptation and (2) modulate cortico-spinal excitability in a way that is specific to the overestimation or underestimation nature of the error.

\section{Method}

\subsection{Video stimuli}

A $66 \mathrm{~cm}$ screen monitor at a resolution of $1024 \times 768$ was used to display a short video to participants, depicting five different actors [3 male, 2 female, mean age $=24.6$ years \pm (SD) .9] repeatedly lifting a small cube $(5 \mathrm{~cm} \times 5 \mathrm{~cm} \times 5 \mathrm{~cm})$ and large cube $(10 \mathrm{~cm} \times 10 \mathrm{~cm} \times 10 \mathrm{~cm})$ in alternation. Unbeknownst to the participants (or the actors in the videos), the cubes had been adjusted to have identical weights (700 g). These stimuli typically elicit the size-weight illusion, along with a stereotyped pattern of fingertip force rate errors during initial lifts (i.e., excessive force for the large cube, and insufficient force for the small cube), followed by a rapid adaptation of fingertip force rates to the actual, and identical, weights of the cubes (see Buckingham \& Goodale, 2010b for details).

Two types of video montages were created (Fig. 1): EV and NEV. We quantified the videos' kinematics by calculating the average load phase duration (averaged from only four actors, as the liftoff data from a 5th actor was lost due to collection errors). Load phase duration was defined as the time which elapsed between the initial application of load force to the object-mounted force transducers (when the force reached a threshold of $>10 \%$ of the maximum overall value) and the point of object liftoff (as measured by liftoff pad detailed below). The EV montages were comprised solely of repeats of actors' first lifts of the small and large cubes (Fig. 1). The other video montage - the NEV - was built up from repeated presentations of same actors' 8th lift of each cube, well-practiced lifts where the actors' fingertip forces were adapted to the actual (identical) weight of the objects (Fig. 1). The actors were unaware of the cubes' adjusted equal weights, and the lifting dynamics in these videos were completely natural and not coached.

In spite of the presence or absence of lifting errors, the EV and NEV montages were visually very similar (see Supplementary Videos 1 and 2). All videos were recorded from the actors' left sides, and showed the inner right forearms of the actors as they gripped the handle and lifted each cube. Each of these videos was approximately 2 min long, and contained a total of 20 lifts. To counter potential order effects with regard to which cube was lifted first, two variants of the EV and NEV montages were created - one where the large cube was lifted before the small cube (watched by half of the participants), and another where the small cube was lifted before the large cube (watched by the other half of the participants).

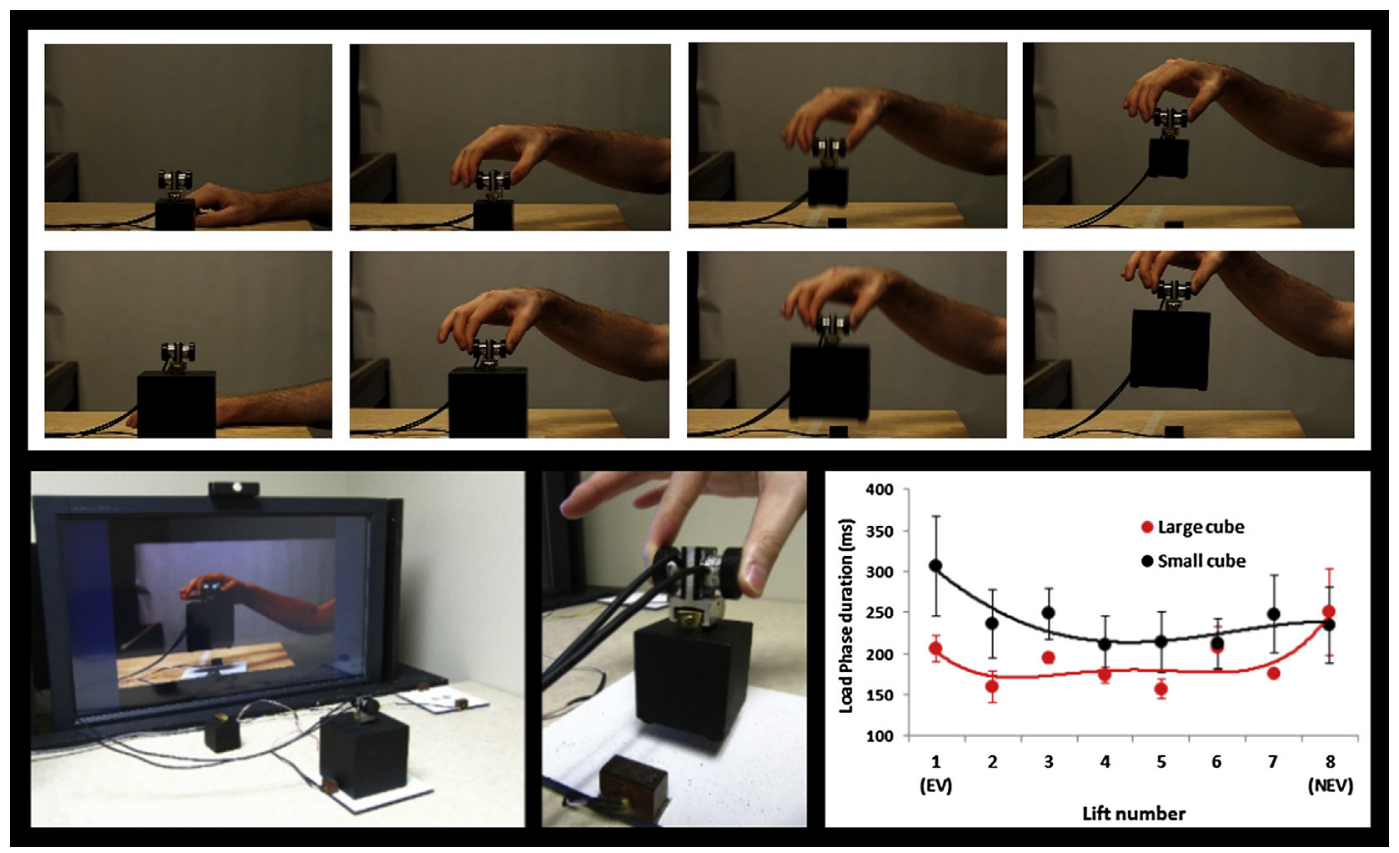

Fig. 1 - The videos watched by participants in both tasks (top panel), the experimental setup for the behavioural task (lower left panel and middle panel). The lower right panel shows the average loading phase durations of the actors lifting in the videos. The error video comprised a montage of lifts from the 1st trial, whereas the no error video comprised a montage of lifts from the 8th trial. 
Supplementary video related to this article can be found at http://dx.doi.org/10.1016/j.cortex.2013.07.004.

\subsection{Behavioural experiment - lifting after observing others' lifts}

Forty-four self-reported right-handed students from the University of Western Ontario took part in the behavioural experiment. Three participants were removed as outliers (with the force rates on multiple trials $>2 \mathrm{SD}$ above the mean), leaving a sample of 41 [6 male, 35 female; mean age $=20.2$ years \pm (SD) 2.3]. Participants had normal or corrected-to normal vision and were naïve to the experimental hypothesis. Testing procedures were approved by the University of Western Ontario Research Ethics Board, and prior to testing, all participants gave written informed consent.

Fingertip forces were measured by a small handle with opposing grip pads that facilitated a precision grip with the thumb and forefinger, which contained a pair of six-axis force-torque sensors (Nano17 F/T; ATI Industrial Automation, Garner, NC). To minimize the possibility of slippage during a lift, the surface of the grip pads were covered with rough sandpaper to provide friction. The cube was placed on a liftoff pad on the table in front of the subject. The pad contained light sensors that projected a small beam of light $3 \mathrm{~mm}$ above and parallel to the surface of the table. These sensors provided a time stamp for object liftoff: when the cube was stationary on the table, the beam of light was broken; when the cube was lifted, the beam of light was unbroken.

This experiment consisted of two stages: an observation stage and a lifting stage. In the observation phase, participants sat on a height-adjustable chair in front of a table and watched one of the videos described above. As lifters' fingertip forces have been shown to rapidly adapt with these stimuli (Buckingham \& Goodale, 2010b), this task utilized a betweenparticipants design. Thus, participants were randomly assigned to either the EV group $(n=21)$ or NEV group $(n=20)$. Again, the video presentation and lifting order (i.e., whether the large cube was lifted before or after the small cube) was counterbalanced across the groups, and congruent within participants (participants who watched the small cube being lifted first, themselves lifted the small cube first).

In the lifting stage of the experiment (i.e., after watching the video), participants simply lifted the cubes that they had just watched the actors lift in the video. Participants wore opaque LCD shutter goggles to ensure that they received no clues as to the cubes' weights between trials. Trials were initiated with a computer-generated auditory cue, at which point the goggle lenses became transparent and the participants gripped the handle with the thumb and forefinger of the right hand. Just as they had seen the actors do in the video, participants lifted the cube approximately $5 \mathrm{~cm}$ off the table in a smooth, controlled fashion and held it steady at the peak of the lift. Four seconds later, a second auditory tone signalled the end of the lift, and participants gently lowered the cube to the liftoff pad. These procedures were repeated, alternating between lifts of the small and large cubes on a trial-by-trial basis for a total of 30 lifts (15 lifts of each). Before watching the videos or lifting any of the cubes, participants were given five practice trials using non-experimental stimuli (blue cylinders), to ensure they were lifting in an appropriate fashion.

The force transducers recorded fingertip forces in the $\mathrm{x}, \mathrm{y}$, and $z$ dimensions at $1000 \mathrm{~Hz}$. The average of the forces tangential to the surface of the grasp pads at each time point was defined as the grip force, whereas the sum of the remaining forces (consisting mostly of those forces opposing gravity) at each time point was defined as the load force. The rates of change of these values were calculated with a 5-point central difference equation, and the peak value was taken to represent our primary dependent variable of sensorimotor prediction peak load force rate (LFR). Additionally, to determine whether lifting observation influenced individuals' perceptions of heaviness we examined the expected weight before, and perceived heaviness after participants had lifted the cubes. To this end, after watching the video, participants gave a number between one (lightest) and one hundred (heaviest) representing how much they expected each cube to weigh. Then, after the lifting phase of the experiment, participants used the same scale to assign a number to how heavy each cube felt to them. All statistical analyses (outlined in the various results sections) were performed with IBM SPSS Statistics version 20 .

\subsection{TMS experiment - MEPs evoked during passive observation of lifts}

Nineteen self-reported right-handed staff and students from the University of Western Ontario took part in the TMS experiment. One participant was removed due to difficulties in reliably localising their hand area, leaving a sample of 18 [12 male, 6 female; mean age $=27.4$ years \pm (SD) 7.0]. Participants had normal or corrected-to normal vision and were naïve to the experimental hypothesis. None of the participants in the TMS experiment has taken part in the earlier behavioural experiment. Testing procedures were approved by the University of Western Ontario Research Ethics Board, and prior to testing, all participants gave written informed consent.

Cortical stimulation was applied with a Magstim air-cooled double $70 \mathrm{~mm}$ (Figure 8) coil via Magstim Rapid 2 stimulator. EMG activity was recorded using surface electrodes (Delsys). Electrodes consisted of three $1 \times 10 \mathrm{~mm}$ parallel silver bars placed $10 \mathrm{~mm}$ apart, which were housed in a compact case containing a $10 \times$ preamplifier. Electrodes were placed to record the activity of the key muscle groups involved in a one-handed precision grip and lift: the right hand adductor pollicis brevis (ADPB) and flexor pollicis brevis (FPB), the wrist flexor (WF), and wrist extensor (WE) muscle groups. The skin around these muscles was cleaned and abraded with alcohol, and the electrodes were attached with adhesive backing and, where necessary, medical tape. Electrode placement was verified using a number of test manoeuvers including movement and isometric force tasks (Gribble \& Ostry, 1998). EMG signals were amplified by a factor of 1000 and digitally sampled at $4000 \mathrm{~Hz}$. The 'hand-knob' region of the left primary motor cortex (M1) was localized based on anatomical landmarks in each individual with a previously-acquired anatomical 3 T Siemens MRI scan, and the focal TMS was guided using Brainsight (Rogue Research Inc.). The TMS was applied focally to the hand region of M1 to evoke MEPs which were measured from the four muscles outlined above. In the setup phase, the experimenters 
refined the stimulation location by grid-searching around the anatomically-localized hand-knob region (average XYZ Talairach coordinates: $-30.3,-29.6,55.7)$ until a cortical area was identified which elicited a visible twitch in the participants hands and consistent MEPs in the relevant hand and arm muscles (average XYZ Talairach coordinates: $-50.9,-24.5$, 73.9). The intensity of the stimulator was then reduced until a TMS pulse elicited no movement, but continued to elicit a visible MEP (i.e., easily distinguishable from baseline in a graphic plot of the electrode activity for $500 \mathrm{msec}$ after the TMS pulse was triggered) in any of the recorded muscles on 7 out of 10 stimulations while at rest.

In this task, which aimed to replicate the general procedure of Alaerts, Senot, et al. (2010), Alaerts, Swinnen, et al. (2010), participants watched the videos while receiving single-pulse TMS over the hand area of the left M1. Participants sat in front of the video monitor with their head in a chin rest and their arm relaxed on the table in a partially supine posture. Single-pulse TMS was applied at a random point during the lift by one of the experimenters pressing a foot pedal. This experimenter stood behind the participant watching the video, to ensure a pulse was applied on each observed lift. A second experimenter, who was blind to the experimental condition, held the coil unsupported over the region identified in the setup phase (i.e., the hand-knob region of M1). Each participant viewed the same EV and NEV montages two times, with the presentation order counterbalanced across participants. As each video contained 20 lifts, participants received 80 TMS pulses over the course of the experimental trials. The signals from the electrodes over the first $250 \mathrm{msec}$ after the TMS pulse (a broad window containing any MEPs) were stored on an external laptop for offline analyses. MEPs with a peak value $>2$ SD above the mean within each subject were defined as outliers and removed. These signals were amplified, bandpass filtered between 10 and $500 \mathrm{~Hz}$, rectified, and then normalized to be a proportion of each individual subject's highest MEP for each individual muscle.

\section{Results}

\subsection{Behavioural experiment - lifting after observing others' lifts}

After watching the video, but prior to actually lifting the cubes, participants verbally reported that they expected the large cube to weigh more than the small cube (Table 1). This expectation did not differ between the EV and NEV groups (Table 1), indicating that participants gained no conscious awareness of the cubes' identical weights from the videos alone. After the lifting portion of the experiment was completed, participants experienced a robust size-weight illusion, reporting that the small cube felt heavier than the large cube (Table 1). Therefore, as with the initial expectations of heaviness, the magnitude of the illusion was similarly unaffected by whether participants had watched the EV or the NEV. Neither the pre-liftoff expectations nor the post-lifting ratings of heaviness correlated with the average LFR or the LFR applied on the first trial for each object in either group (Supplementary Table 1).

In contrast to the cognitive measures, a clear difference emerged between the sensorimotor predictions made by the EV and NEV groups when they lifted the large and small cubes. Participants who watched the NEV lifted the cubes initially with the usual pattern of overestimations and underestimations seen for these objects (e.g., Buckingham \& Goodale, 2010b). After a few trials, however, and in line with previous research (Flanagan \& Beltzner, 2000) these errors were rapidly corrected, with the lifting forces rapidly reaching an asymptote (Fig. 2A). In contrast, participants who watched the EV appeared to require very little adaptation of their forces when lifting the large cube - they lifted the large cube with approximately the same rate of force throughout the entire experiment (Fig. 2B). In other words, participants who watched the EV made almost no overestimation-style errors. In order to quantify the benefit that is gained from watching the EV over watching the NEV, we created a simple metric of the total amount of fingertip force adaptation required for each cube in each condition over the course of the experiment by calculating the difference between the force rates on trial 1 (the initial error) and trial 15 (the final and presumably most well-adapted value). First, we examined this values in a mixed-design 2 (cube size) $\times 2$ (video) ANOVA, and followed this omnibus test up with post hoc Bonferroni-corrected independent samples $t$ tests. From the ANOVA, we observed a significant main effect of size $[F(1,38)=33.20, p<.001]$ and a significant interaction between video and size $[F(1,38)=6.06$, $p<.05]$. Post hoc analyses confirmed that, although there were no differences between the groups with regard to their lifts of the small cube $[t(39)=.12, p=.91 ;$ Fig. $2 C]$, the EV group did indeed outperform the NEV group when lifting the large cube $[\mathrm{t}(39)=2.57, p<.05 ;$ Fig. $2 \mathrm{C}]$. Thus, participants were, to a

Table 1 - Participants' expectations of heaviness before lifting and their perceptions of heaviness after lifting in the behavioural experiment, as a function of the size of the cube they were lifting (the within-subject comparison) and the video they watched (the between-subject comparison).

\begin{tabular}{|c|c|c|c|c|c|c|}
\hline & \multicolumn{2}{|c|}{$\begin{array}{c}\text { Expected heaviness } \\
\text { before lifting } \\
\text { (mean } \pm \text { standard error) }\end{array}$} & \multirow[t]{2}{*}{$\begin{array}{l}\text { Within-subject } \\
\text { t tests } \\
\text { (large us small) }\end{array}$} & \multicolumn{2}{|c|}{$\begin{array}{c}\text { Perceived heaviness } \\
\text { after lifting } \\
\text { (mean } \pm \text { standard error) }\end{array}$} & \multirow[t]{2}{*}{$\begin{array}{c}\text { Within-subject } \\
\quad t \text { tests } \\
\text { (large us small) }\end{array}$} \\
\hline & Large cube & Small cube & & Large cube & Small cube & \\
\hline Error video & $63.1 \pm 3.3$ & $37.7 \pm 5.3$ & $p=.001$ & $41.7 \pm 4.7$ & $65.6 \pm 3.6$ & $p<.001$ \\
\hline No error video & $59.8 \pm 5.0$ & $32.3 \pm 5.4$ & $p=.003$ & $31.7 \pm 4.7$ & $60.3 \pm 4.6$ & $p<.001$ \\
\hline $\begin{array}{l}\text { Between subject } t \text { tests } \\
\text { (Error video versus no error video) }\end{array}$ & $p=.57$ & $p=.48$ & & $p=.14$ & $p=.37$ & \\
\hline
\end{tabular}



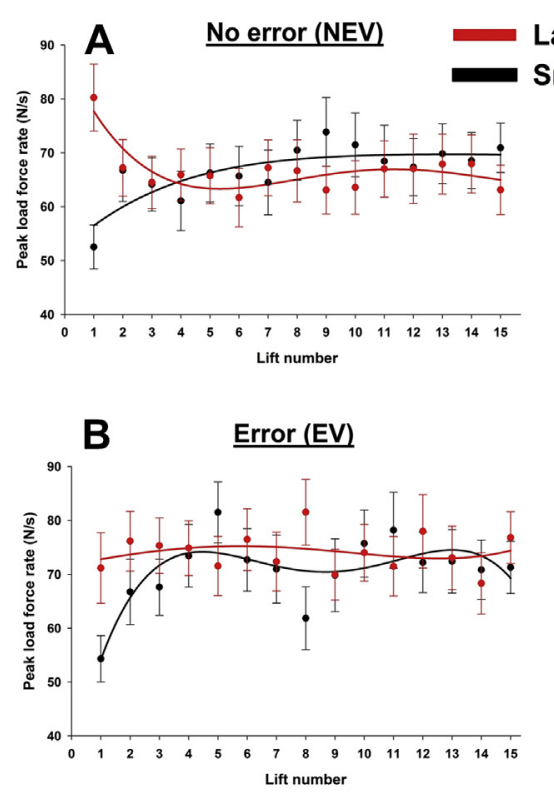

Large cube

Small cube

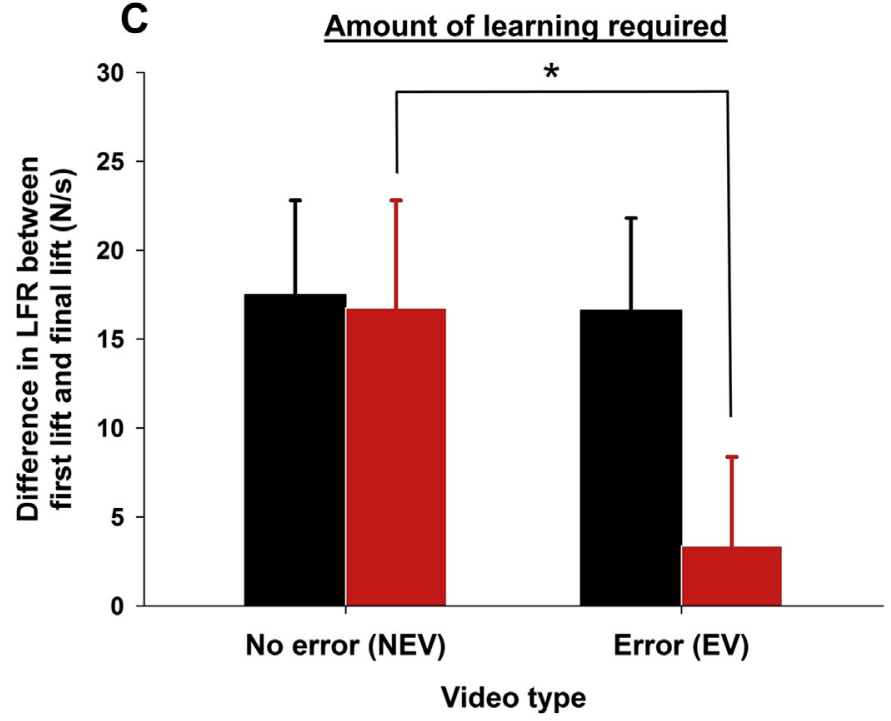

Fig. 2 - The peak LFRs used to lift the identically weighted the large and small cubes on each trial for (A) the no error video group and (B) the error video group. These data are fit with a 4th order polynomial to better visualize the linear trends, and error bars show between-participants standard error of the means. The differences between the error video and no error video groups $(C)$ were quantified by comparing the amount of adaptation that took place across the entire experiment (as indexed by the difference between the initial force errors on trial 1 and the final adapted forces on trial 15). Data are plotted on the positive axis for overestimations and underestimations. ${ }^{*}$ indicates an alpha of .05 .

degree, able to learn from the mistakes of others to improve their lifting performance.

\subsection{TMS experiment - MEPs evoked during passive observation of lifts}

We followed-up our behavioural experiment with a TMS study to determine the neural effects of observing the error-filled and error-free video stimuli. In this task, cortico-spinal excitability during the observation of these lifts was determined by examining the magnitude of MEPs elicited by TMS in observers who were watching lifts of identically weighted large and small cubes. In the EV condition, participants watched actors overestimating the weight of the large cube and underestimating the weight of the small cube; in the NEV condition, participants observed actors lifting the cubes with identical forces. For our initial exploratory analysis, we examined the MEPs from four hand and arm muscles in a MANOVA for the condition most comparable to the prior object lifting observation studies - comparing the NEV lifts of the large and small cubes (Alaerts et al., 2009). In this omnibus test we noted significantly higher MEPs when participants observed lifts of the large cube as compared to when they watched lifts of the small cube $[F(1,17)=6.07, p<.05]$. Of the four muscles recorded from, inspection of the data showed that this size-based cortico-spinal modulation was driven largely by the ADPB, shown in Fig. 3 (details of electrode positioning and MEP magnitudes for all muscles' can be found in the Methods and Supplementary Fig. 1, respectively). To examine the modulatory effects in the ADPB alone, the large and small cube MEP magnitudes in the various conditions were examined in a 2 (cube size) $\times 2$ (video type) ANOVA with repeated measures. As with the multivariate test, there were no main effects of video condition $[F(1,17)=.4, p=.53]$ or cube size $[F(1,17)=1.54, p=.23]$. There was, however, an indication of an interaction between video condition and cube size that did not reach statistical significance $[F(1,17)=3.47, p=.08]$,

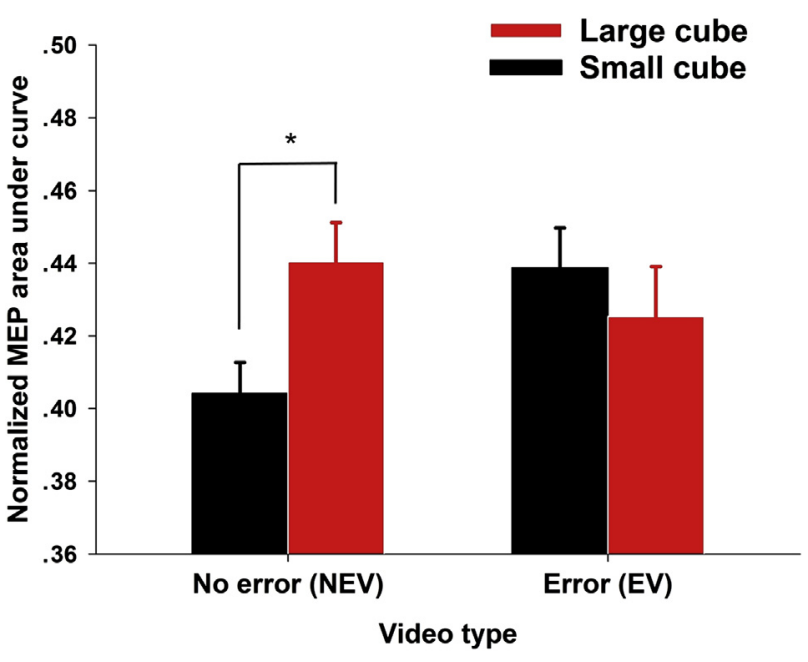

Fig. 3 - The normalized MEP area under the curve for the MEPs recorded from the ADPB while watching error and no error lifts of the identically weighted small and large cubes. Error bars show between subject standard error of the means. * indicates an alpha of .05. Error bars show normalized within-subject standard error of the means for each condition. 
which we examined with Bonferroni-corrected pairedsample post hoc $t$ tests. These analyses confirmed that, when observing lifts that did not contain errors (NEV condition), participants' MEPs were significantly larger when observing the large cube than when observing the small cube $[t(17)=3.02, p<.05$, Fig. 3A]. However, this 'size effect' was completely eradicated when watching errors $[t(17)=.69$, $p=.50$; Fig. 3B], and there was no hint of any sizebased modulation of cortico-spinal activity for watching overestimations of the large cube as compared to underestimations of the small cube. As there were no differences in the background EMG between any of the conditions (all $p$ values $>$.17, see Supplementary Fig. 2), these effects are likely to have been caused by differences in cortio-spinal excitability induced by the various observation conditions.

\subsection{General discussion}

In the current work, we examined the effect that the observation of object lifting errors has on individuals' lifting performance and cortico-spinal excitability. Separate groups of participants watched videos of actors lifting the identically weighted large and small wooden cubes that typically elicit size-weight illusions and fingertip force errors. One (the EV) showed naïve actors making typical overestimation and underestimation-style errors made when lifting these cubes, and the other (the NEV) showed the same naïve actors lifting the cubes with very similar, well-adapted forces. Participants who observed both of the cubes being lifted with the same force as one another (i.e., without errors - NEV) tended to lift the cubes with incorrect fingertip forces (i.e., excessive force for the large cube and less force for the small cube). Participants who observed the error-filled lifts (EV), however, were far less prone to overestimating the weight of the large cube than their counterparts, suggesting they were learning through observing these errors. We also examined corticospinal excitability when another group of participants who passively observed error-filled and error-free lifts. When participants observed the cubes being lifted with the same force as one another (i.e., without errors), a robust size-based cortico-spinal modulation was evident: larger MEPs were elicited when participants observed lifts of the large cube than when they observed lifts of the smaller cube. When participants watched these same cubes being lifted with fingertip force errors, however, this size-based cortico-spinal modulation was completely eradicated; approximately equal magnitude MEPs were elicited whether participants watched errors based on overestimations of the large cube or errors based on underestimations of the small cube. In isolation, the behavioural and cortico-spinal findings make substantial contributions to their respective literature, and together may point toward a low-level mechanism linking observational learning to fingertip force adaptation.

In our behavioural experiment, participants who watched the EV made significantly fewer overestimation-style errors than participants who watched the NEV. In short, the current study is a striking demonstration of the powerful effect that information derived through visual observation can have on a task that is heavily dependent on haptic feedback (Johansson \& Flanagan, 2009). Not only do these findings highlight the sensitivity of the human sensorimotor system to subtle visual cues (Buckingham et al., 2011), they also highlight the stark differences between the sensorimotor system and conscious expectations of heaviness. Neither the EV nor the NEV groups gained any conscious insight into the actual (identical) weight of the cubes from watching the videos, and their subsequent size-weight illusion was similarly unaffected. The fact that only the observers' LFR scaling was influenced by watching others lift further suggests a low-level, non-cognitive mechanism, is underpinning these behavioural findings. These behavioural findings confirm and extend the idea that separate internal representations underpin the way we lift objects and the way we experience how heavy they feel (Chouinard, Large, Chang, \& Goodale, 2009; Flanagan \& Beltzner, 2000; Flanagan, Bittner, \& Johansson, 2008).

It is worth taking time to discuss why those who watched the EVs outperformed their counterparts when lifting the large cube but not the small cube. We suspect that this interesting difference between overestimations and underestimations stems from the reliability of information available when watching the kinematic consequences of overestimations. Overestimation errors are characterized by rapid movements, large accelerations, and extremely short loading phase durations - kinematics that happen to be particularly visually salient. Furthermore, the load phase duration of actors' overestimation-style lifts had a far lower standard deviation than any of the other lifts which participants viewed; in other words, these lifts were more consistent. This relative saliency and consistency could allow the appearance and magnitude of an overestimation error to be readily identified and subsequently corrected. Underestimations of forces are, by contrast, defined by a mismatch between the expected and actual liftoff time (Johansson \& Flanagan, 2009), which merely results in the object not moving. As an unmoving object is not a de facto cue to error, and as observers do not know when the actors expected liftoff to occur, underestimations may not have been consistently identified as errors, and consequently were not utilized to pre-adapt the lifts of the small cube. Future work could directly assess this proposition by examining fingertip forces after explicitly informing observers about the errorfilled or error-free content of the videos they are observing.

In contrast to the straightforward outcomes of the behavioural experiment, our study examining cortico-spinal excitability during passive observations of lifts yielded a more complex pattern of results. We shall first examine the implications of the MEP data in the NEV condition in the context of the lifting observation studies undertaken by Alaerts and colleagues (Alaerts, Senot, et al., 2010, Alaerts, Swinnen, et al., 2010, Alaerts et al., 2009). These authors demonstrated that observing error-free lifts of heavy-looking stimuli evokes larger MEP than observing lifts of light-looking stimuli. Subsequent research demonstrated that this effect appears to be driven by kinematic differences in the way that light and heavy objects are lifted, rather than differences in how heavy they look simply on the basis of their apparent static weight (Alaerts et al., 2009; Senot et al., 2011). In the current work, when comparing the EV to the NEV when object size was held constant, we noted that the EV and the NEV elicited similar MEPs for the small cube $[t(17)=.66, p=.52]$. There was a trend 
for the EV to elicit a smaller MEP than the NEV when watching lifts of the large cube, but this comparison did not reach statistical significance $[\mathrm{t}(17)=1.99, p=.06]$. In contrast, the current work appears to demonstrate that object size has a greater effect on cortico-spinal excitability than object kinematics: when participants in our experiment observed the NEV condition, they showed a greater level of cortico-spinal excitability watching lifts of a large cube as compared to when they watch lifts of an equally weighted small cube even though the kinematics of these lifts were virtually identical (see Materials and Methods section). It is possible that the discrepancies between our work and the findings of Alaerts et al. (2009) may stem from differences in how the optimal hand area in primary motor cortex was defined between the studies.

This departure from prior literature may be due to the fact that participants observed actors performing lifts in the context of a situation in which object size was the only (misleading) cue to weight. Previous attempts to disentangle static and kinematic visual cues to weight have manipulated either actual object mass or a variety of different visual cues to weight (e.g., the presence or absence of a visible weight, the amount of water/sand in a bottle, or the label on an object); to our knowledge, our MEP task is the only action observation study to manipulate the visual size of the object. We suspect that this novel size-based cortico-spinal modulation stems from the reliability of volume, as inferred by visual size, as a cue to weight in our environment. This 'size effect' is consistent with our previous suggestions that visual size may be such a powerful cue to object weight that stimulus volume may have an automatic modulatory effect on the sensorimotor system (Buckingham \& Goodale, 2010b). It remains to be seen whether other cues to weight (e.g., material) have similar low-level modulatory effects.

It is within the context of the size-based cortico-spinal modulations that we must examine the MEPs which were elicited when viewing errors. There was no difference between the magnitudes of the MEPs elicited when participants watched lifts of large and small cubes in the EV condition, suggesting that the typical cortico-spinal modulations elicited when viewing error-free lifts were eradicated. To reconcile our data with findings of past research on this topic, we suggest that a different, opposing, effect is counteracting the usual size-based modulation of cortico-spinal excitability that has been observed with NEV. Theoretically, this effect could have arisen from differences in the actors' kinematics in the EV when they lifted the large cubes as compared to when they lifted the small cubes. From the perspective of an observer, overestimations of force tend to result in easier-looking lifting kinematics (i.e., the large cube was lifted with rapid accelerations and short load phase durations). By contrast, the underestimations of force that were applied to the small cube give it the kinematics of a heavier object (see Supplementary Video 1). It has been convincingly demonstrated that observing such easy- and difficult-looking lifting kinematics can in fact modulate cortico-spinal activity such that observing lifts that look effortful will elicit larger MEPs than observing easy-looking lifts (Senot et al., 2011). It is likely that these kinematic-based modulations in cortico-spinal excitability oppose the size-based effects seen in the NEV, leading to the similar MEPs when watching error-filled lifts of large and small cubes (see also Obhi \& Hogeveen, 2010).

Our MEP findings demonstrate that the cortico-spinal excitability during observation of a lift is a joint function of visual information about (1) the size of the object to be lifted, and, if available, (2) the weight of an object gleaned from observing kinematics of an individual lifting the same object. These ideas are consistent with the additive combination mechanism which has been proposed by (Loh, Kirsch, Rothwell, Lemon, \& Davare, 2010), who demonstrated that an individual's pre-existing internal models of heaviness (i.e., sensorimotor memories from previous lifts) are combined with static visual cues to object mass prior to lifting - a process which evolves over a course of several hundred milliseconds. Our findings argue for the inclusion of observed kinematic information from prior lifts (evidently a reliable source of possible weight information for the sensorimotor system in this simple model). In support of this idea, Alaerts, de Beukelaar, Swinnen, and Wenderoth (2011) have shown that, in a blocked design, visual information about object weight from previously observed lifts drives cortico-spinal excitability before visual information about object weight in an upcoming observed lift is available. These low-level modulations may even serve a behavioural function, given the growing experimental evidence for a link between cortico-spinal excitability and motor output (Bagce, Saleh, Adamovich, Krakauer, \& Tunik, 2013; Klein-Flügge, Nobbs, Pitcher, \& Bestmann, 2013; Orban de Xivry, Ahmadi-Pajouh, Harran, Salimpour, \& Shadmehr, 2013). In the context of object lifting, Loh et al. (2010) have provided strong evidence that cortico-spinal excitability is related to fingertip force scaling by demonstrating that, in the same individuals, the ratio between MEPs before lifting for heavy and light objects correlates with the ratio for the force rates used to lift the same heavy and light objects. Thus, the cortico-spinal consequences of observing an error (even an error made by oneself) could automatically drive the correction of that error for future lifts, contributing to processes underpinning fingertip force adaptation and perhaps motor learning in general. If so, observation of one's own actions may a critical cue for errorbased learning, possibly accounting for our recent findings that lifting without visual feedback impairs fingertip force adaptation (Buckingham \& Goodale, 2010a; Buckingham et al., 2011). Thus, one role of the sensorimotor system is to gather evidence about the likely requirements of an upcoming motor plan from a variety of sources to drive successful behaviour. Although the current dataset is not able to directly speak to this question, as the TMS pulses were not time-locked to kinematic events in the video. we hope to explicitly test this prediction in future research by measuring lifting behaviour and cortico-spinal excitability at various time points throughout montages of observed lifts, when only a fraction of the kinematic error information is available (cf. Alaerts et al., 2011).

The current findings also provide an interesting new take on theories regarding the 'action observation system', where motor skills activate the same neural circuitry in left premotor cortex as observing the same action (Malfait et al., 2010; Mukamel et al., 2010). This link is normally considered to be an automatic mirroring relationship, implicitly preparing the 
observer to mimic the observed act. Our cortico-spinal and behavioural findings both point toward an automatic link between observation of an action and sensorimotor output. However, our findings clearly refute the suggestion that the sensorimotor system simply mirrors observed behaviour (Meulenbroek et al., 2007). Rather, our findings point toward a more complex relationship between the observer's prior knowledge and the dynamics of the observed task in relation to their own kinematic experiences. An observer's perceptual expertise with the visual kinematics of biological motion combines with their prior expectations of how heavy and light objects are usually lifted to provide a context for the observed action. In the case of object lifting, when stimulus properties are observed in conjunction with incompatible lifting kinematics (e.g., a heavy-looking object being lifted with lightlooking kinematics), the relationship between observation and behaviour becomes contrastive rather than integrative (Hamilton et al., 2004; Meulenbroek et al., 2007). It is feasible that, across a wide range of actions, (1) a lifetime's worth of visual experience of one's own kinematics and (2) an understanding of the statistics of the environment allows observed movements to be categorized as optimal or suboptimal, driving subsequent behaviour.

To sum up, this work has demonstrated that (1) observing object lifting errors improves subsequent object lifting performance when compared to observing error-free performance, and (2) this behavioural improvement is accompanied by sensorimotor modulations at the level of cortico-spinal excitability when observing these lifts. Not only do these findings shed light on the interactions that occur prior to sensorimotor prediction in the context of object lifting, but they pose larger questions about how we can best learn new motor skills. Extending the current findings to the topic of motor learning in general, one might predict that those who are learning new skills could profit more from watching others make mistakes than they would from watching experts - a conclusion which has exciting implications for the teaching of visuo-motor skills.

\section{Acknowledgements}

The authors would like to thank J. Ladich for stimulus construction as well as $\mathrm{H}$. Yang for technical support. We would also like to thank J. Paciocco for assistance with Brainsight as well as L. Strother for helpful comments on an earlier draft of the manuscript. Finally, we would like to thank the actors who featured in the video stimuli. G. Buckingham was supported with a Banting Postdoctoral Fellowship, awarded by the Natural Sciences and Engineering Council of Canada (NSERC). The funders had no role in study design, data collection and analysis, decision to publish, or preparation of the manuscript. The authors have declared that no competing interests exist.

\section{Supplementary data}

Supplementary data related to this article can be found at http://dx.doi.org/10.1016/j.cortex.2013.07.004.

\section{R E F E R E N C E S}

Alaerts, K., de Beukelaar, T. T., Swinnen, S. P., \& Wenderoth, N. (2011). Observing how others lift light or heavy objects: timedependent encoding of grip force in the primary motor cortex. Psychological Research, 76(4), 503-513.

Alaerts, K., Senot, P., Swinnen, S. P., Craighero, L., Wenderoth, N., \& Fadiga, L. (2010a). Force requirements of observed object lifting are encoded by the observer's motor system: a TMS study. European Journal of Neuroscience, 31(6), 1144-1153.

Alaerts, K., Swinnen, S. P., \& Wenderoth, N. (2009). Is the human primary motor cortex activated by muscular or directiondependent features of observed movements? Cortex, 45(10), $1148-1155$

Alaerts, K., Swinnen, S. P., \& Wenderoth, N. (2010b). Observing how others lift light or heavy objects: which visual cues mediate the encoding of muscular force in the primary motor cortex? Neuropsychologia, 48(7), 2082-2090.

Bagce, H. F., Saleh, S., Adamovich, S. V., Krakauer, J. W., \& Tunik, E. (2013). Corticospinal excitability is enhanced after visuomotor adaptation and depends on learning rather than performance or error. Journal of Neurophysiology, 109(4), 1097-1106.

Bingham, G. P. (1987). Kinematic form and scaling: further investigations on the visual perception of lifted weight. Journal of Experimental Psychology. Human Perception and Performance, 13(2), 155-177.

Brown, L. E., Wilson, E. T., \& Gribble, P. L. (2009). Repetitive transcranial magnetic stimulation to the primary motor cortex interferes with motor learning by observing. Journal of Cognitive Neuroscience, 21(5), 1013-1022.

Brown, L. E., Wilson, E. T., Obhi, S. S., \& Gribble, P. L. (2010). Effect of trial order and error magnitude on motor learning by observing. Journal of Neurophysiology, 104(3), 1409-1416.

Buckingham, G., Cant, J. S., \& Goodale, M. A. (2009). Living in a material world: how visual cues to material properties affect the way that we lift objects and perceive their weight. Journal of Neurophysiology, 102(6), 3111-3118.

Buckingham, G., \& Goodale, M. A. (2010a). Lifting without seeing: the role of vision in perceiving and acting upon the size weight illusion. PLOS ONE, 5(3), e9709.

Buckingham, G., \& Goodale, M. A. (2010b). The influence of competing perceptual and motor priors in the context of the size-weight illusion. Experimental Brain Research, 205(2), 283-288.

Buckingham, G., Ranger, N. S., \& Goodale, M. A. (2011). The role of vision in detecting and correcting fingertip force errors during object lifting. Journal of Vision, 11(1).

Calvo-Merino, B., Glaser, D., Grèzes, J., Passingham, R., \& Haggard, P. (2005). Action observation and acquired motor skills: an FMRI study with expert dancers. Cerebral Cortex, 15(8), 1243-1249.

Chouinard, P., Large, M., Chang, E., \& Goodale, M. (2009). Dissociable neural mechanisms for determining the perceived heaviness of objects and the predicted weight of objects during lifting: an fMRI investigation of the size-weight illusion. NeuroImage, 44(1), 200-212.

Flanagan, J. R., \& Beltzner, M. A. (2000). Independence of perceptual and sensorimotor predictions in the size-weight illusion. Nature Neuroscience, 3(7), 737-741.

Flanagan, J. R., Bittner, J. P., \& Johansson, R. S. (2008). Experience can change distinct size-weight priors engaged in lifting objects and judging their weights. Current Biology, 18(22), 1742-1747.

Gallese, V., Gernsbacher, M. A., Heyes, C., Hickok, G., \& Iacoboni, M. (2011). Mirror neuron forum. Perspectives on Psychological Science, 6(4), 369-407. 
Gordon, A. M., Forssberg, H., Johansson, R. S., \& Westling, G. (1991). Visual size cues in the programming of manipulative forces during precision grip. Experimental Brain Research, 83(3), 477-482.

Grandy, M. S., \& Westwood, D. A. (2006). Opposite perceptual and sensorimotor responses to a size-weight illusion. Journal of Neurophysiology, 95(6), 3887-3892.

Gribble, P. L., \& Ostry, D. J. (1998). Independent coactivation of shoulder and elbow muscles. Experimental Brain Research, 123(3), 355-360.

Hamilton, A., Wolpert, D., \& Frith, U. (2004). Your own action influences how you perceive another person's action. Current Biology, 14(6), 493-498.

Hamilton, AF. de C., Joyce, D. W., Flanagan, J. R., Frith, C. D., \& Wolpert, D. M. (2007). Kinematic cues in perceptual weight judgement and their origins in box lifting. Psychological Research, 71(1), 13-21.

Jeannerod, M. (2001). Neural simulation of action: a unifying mechanism for motor cognition. NeuroImage, 14(1), S103-S109.

Johansson, R. S., \& Flanagan, J. R. (2009). Coding and use of tactile signals from the fingertips in object manipulation tasks. Nature Reviews Neuroscience, 10(5), 345-359.

Johansson, R. S., \& Westling, G. (1988). Coordinated isometric muscle commands adequately and erroneously programmed for the weight during lifting task with precision grip. Experimental Brain Research, 71(1), 59-71.

Klein-Flügge, M. C., Nobbs, D., Pitcher, J. B., \& Bestmann, S. (2013). Variability of human corticospinal excitability tracks the state of action preparation. Journal of Neuroscience, 33(13), 5564-5572.

Loh, M. N., Kirsch, L., Rothwell, J. C., Lemon, R. N., \& Davare, M. (2010). Information about the weight of grasped objects from vision and internal models interacts within the primary motor cortex. Journal of Neuroscience, 30(20), 6984-6990.

Malfait, N., Valyear, K. F., Culham, J. C., Anton, J. L., Brown, L. E., \& Gribble, P. L. (2010). fMRI activation during observation of others' reach errors. Journal of Cognitive Neuroscience, 22(7), 1493-1503.

Mattar, A. A. G., \& Gribble, P. L. (2005). Motor learning by observing. Neuron, 46(1), 153-160

Meulenbroek, R. G. J., Bosga, J., Hulstijn, M., \& Miedl, S. (2007). Joint-action coordination in transferring objects. Experimental Brain Research, 180(2), 333-343.

Mon-Williams, M., \& Murray, A. H. (2000). The size of the visual size cue used for programming manipulative forces during precision grip. Experimental Brain Research, 135(3), 405-410.

Mukamel, R., Ekstrom, A. D., Kaplan, J., Iacoboni, M., \& Fried, I. (2010). Single-neuron responses in humans during execution and observation of actions. Current Biology, 20(8), 750-756.

Obhi, S. S., \& Hogeveen, J. (2010). Incidental action observation modulates muscle activity. Experimental Brain Research, 203(2), $427-435$

Orban de Xivry, J.-J., Ahmadi-Pajouh, M. A., Harran, M. D., Salimpour, Y., \& Shadmehr, R. (2013). Changes in corticospinal excitability during reach adaptation in force fields. Journal of Neurophysiology, 109(1), 124-136.

Senot, P., D’Ausilio, A., Franca, M., Caselli, L., Craighero, L., \& Fadiga, L. (2011). Effect of weight-related labels on corticospinal excitability during observation of grasping: a TMS study. Experimental Brain Research, 211(1), 161-167.

Uçar, E., \& Wenderoth, N. (2012). Movement observation affects sensorimotor memory when lifting a familiar object. Cortex, 48(5), 638-640. 